\title{
Minimum Compliance Topology Optimization of Shell-Infill Composites for Additive Manufacturing
}

Wu, Jun; Clausen, Anders; Sigmund, Ole

Published in:

Computer Methods in Applied Mechanics and Engineering

Link to article, DOI:

10.1016/j.cma.2017.08.018

Publication date:

2017

Document Version

Peer reviewed version

Link back to DTU Orbit

Citation (APA):

Wu, J., Clausen, A., \& Sigmund, O. (2017). Minimum Compliance Topology Optimization of Shell-Infill Composites for Additive Manufacturing. Computer Methods in Applied Mechanics and Engineering, 326, 358-375. https://doi.org/10.1016/j.cma.2017.08.018

\section{General rights}

Copyright and moral rights for the publications made accessible in the public portal are retained by the authors and/or other copyright owners and it is a condition of accessing publications that users recognise and abide by the legal requirements associated with these rights.

- Users may download and print one copy of any publication from the public portal for the purpose of private study or research.

- You may not further distribute the material or use it for any profit-making activity or commercial gain

- You may freely distribute the URL identifying the publication in the public portal 


\section{Accepted Manuscript}

Minimum compliance topology optimization of shell-infill composites for additive manufacturing

Jun $\mathrm{Wu}$, Anders Clausen, Ole Sigmund

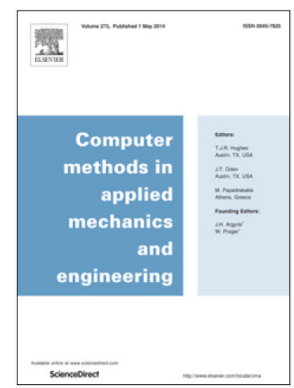

PII:

S0045-7825(17)30598-4

DOI:

http://dx.doi.org/10.1016/j.cma.2017.08.018

Reference: CMA 11559

To appear in: Comput. Methods Appl. Mech. Engrg.

Received date: 17 May 2017

Revised date: 7 August 2017

Accepted date : 11 August 2017

Please cite this article as: J. Wu, A. Clausen, O. Sigmund, Minimum compliance topology optimization of shell-infill composites for additive manufacturing, Comput. Methods Appl. Mech. Engrg. (2017), http://dx.doi.org/10.1016/j.cma.2017.08.018

This is a PDF file of an unedited manuscript that has been accepted for publication. As a service to our customers we are providing this early version of the manuscript. The manuscript will undergo copyediting, typesetting, and review of the resulting proof before it is published in its final form. Please note that during the production process errors may be discovered which could affect the content, and all legal disclaimers that apply to the journal pertain. 


\title{
Minimum Compliance Topology Optimization of Shell-Infill Composites for Additive Manufacturing
}

\author{
Jun $\mathrm{Wu}^{\mathrm{a}, *}$, Anders Clausen ${ }^{\mathrm{b}}$, Ole Sigmund ${ }^{\mathrm{b}}$ \\ ${ }^{a}$ Department of Design Engineering, Delft University of Technology, Delft, The Netherlands \\ ${ }^{b}$ Department of Mechanical Engineering, Technical University of Denmark, Lyngby, Denmark
}

\begin{abstract}
Additively manufactured parts are often composed of two sub-structures, a solid shell forming their exterior and a porous infill occupying the interior. To account for this feature this paper presents a novel method for generating simultaneously optimized shell and infill in the context of minimum compliance topology optimization. Our method builds upon two recently developed approaches that extend density-based topology optimization: A coating approach to obtain an optimized shell that is filled uniformly with a prescribed porous base material, and an infill approach which generates optimized, non-uniform infill within a prescribed shell. To evolve the shell and infill concurrently, our formulation assigns two sets of design variables: One set defines the base and the coating, while the other set defines the infill structures. The resulting intermediate density distributions are unified by a material interpolation model into a physical density field, upon which the compliance is minimized. Enhanced by an adapted robust formulation for controlling the minimum length scale of the base, our method generates optimized shell-infill composites suitable for additive manufacturing. We demonstrate the effectiveness of the proposed method on numerical examples, and analyze the influence of different design specifications.
\end{abstract}

Keywords: Topology optimization, additive manufacturing, two-scale structure, infill, coating, composite

\section{Introduction}

Topology optimization has been recognized as an important design method for additive manufacturing, as it fully leverages the manufacturing flexibility enabled by the layer-upon-layer additive process. It finds an optimized material distribution in the design space to maximize the structural performance under given boundary conditions and constraints [1]. Early works in topology optimization are summarized in the book [2], and recent developments until 2013 are reviewed in [3,4].

While topology optimized material distributions mostly represent solid models, engineering practices in additive manufacturing seem to favour porous structures $[5,6]$. In fused deposition modeling (FDM), a commonly used additive manufacturing technology, the interior of 3D

\footnotetext{
*Corresponding author

Email address: $\mathrm{j}$.wu-1@tudelft.nl (Jun Wu)
} 
models is often represented by repetitive infill patterns (e.g., triangles and hexagons). The porous infill is introduced to control the cost associated with material usage and printing time. The shellinfill composite involves a few parameters, including the shell thickness, infill pattern, and infill volume percentage. These parameters are specified by designers to roughly balance cost and mechanical properties. In general a larger shell thickness and a larger infill volume percentage lead to a stronger print, while consuming more material and prolonging the printing time.

Our current research is motivated from two perspectives. First, post-processing topologically optimized solids into shell-infill composites guarantees no optimality on the final structure, thereby wasting the efforts of the sophisticated numerical optimization. It is thus of high interest to consider such a shell-infill composite directly in the structural optimization routine, eliminating the conversion from optimized solids to sub-optimal shell-infill composites. Second, shell-infill composites can obtain significantly increased stability with respect to buckling [7] and unpredicted loading conditions [8] at the expense of a minor increase in compliance. Given the manufacturing flexibility enabled by additive manufacturing, such two-scale structures have a high potential to be widely employed in industrial metal printing (e.g., using selective laser melting).

Two recent developments (partially) address the optimal design of shell-infill composites by extending density-based topology optimization known as SIMP (Solid Isotropic Material with Penalization) [9]. These two extensions are complementary in the sense that they optimize one component in the composite, i.e., shell or infill, while assuming the other component prescribed. Specifically, Clausen et al. $[10,11]$ proposed a method to design coated structures, i.e., a composition of a solid shell and base material. The base material can be interpreted as a uniform infill, with a homogenized stiffness smaller than the stiffness of the solid coating material. The coatingbase structure is obtained by introducing a two-step filtering process to separate the base and the coating from a scalar field of design variables. Conversely, to optimize infill within a prescribed shell, Wu et al. [8] presented a method to design bone-inspired micro-structures as porous infill. This is achieved by introducing an upper bound on a local volume measure, in order to regulate the local material distribution. The idea of local upper bounds is similar to maximum length scale $[12,13]$. The resulting porous infill is dominated by crossing sub-structures, distributed in the entire space enclosed by the prescribed solid shell, and following principal stress directions. The optimized infill performs much stiffer under given boundary conditions than the commonly used, uniformly repetitive infill patterns.

This paper moves a step further and presents a complete solution to the optimal design of shell-infill composites by concurrently evolving the shell interface and the micro-structural infill. In particular, we propose a novel formulation to consider both the coating-base and infill constraints in density-based topology optimization. Two design fields are utilized to respectively derive the coating-base distribution and the infill distribution. The intermediate distributions are unified by a material interpolation scheme into the final physical density field, based on which the compliance is minimized. Furthermore, the robust formulation [14] is adapted to ensure length scale in the composite, leading to distinct infills.

The design of shell-infill composites is among recent developments addressing geometric constraints for additive manufacturing. Langelaar [15], Qian [16], and Gaynor and Guest [17] proposed methods to ensure the property of self-support in optimized structures. Such methods follow the filtering scheme proposed by Guest et al. [18] and extended in [19, 12, 20] which are also the basis of our current work. Wu et al. [21] proposed a rhombic pattern as a special selfsupport infill, and performed infill optimization by adaptively subdividing the rhombic cells. The length scale problem relevant to manufacturing technologies in general is thoroughly examined 


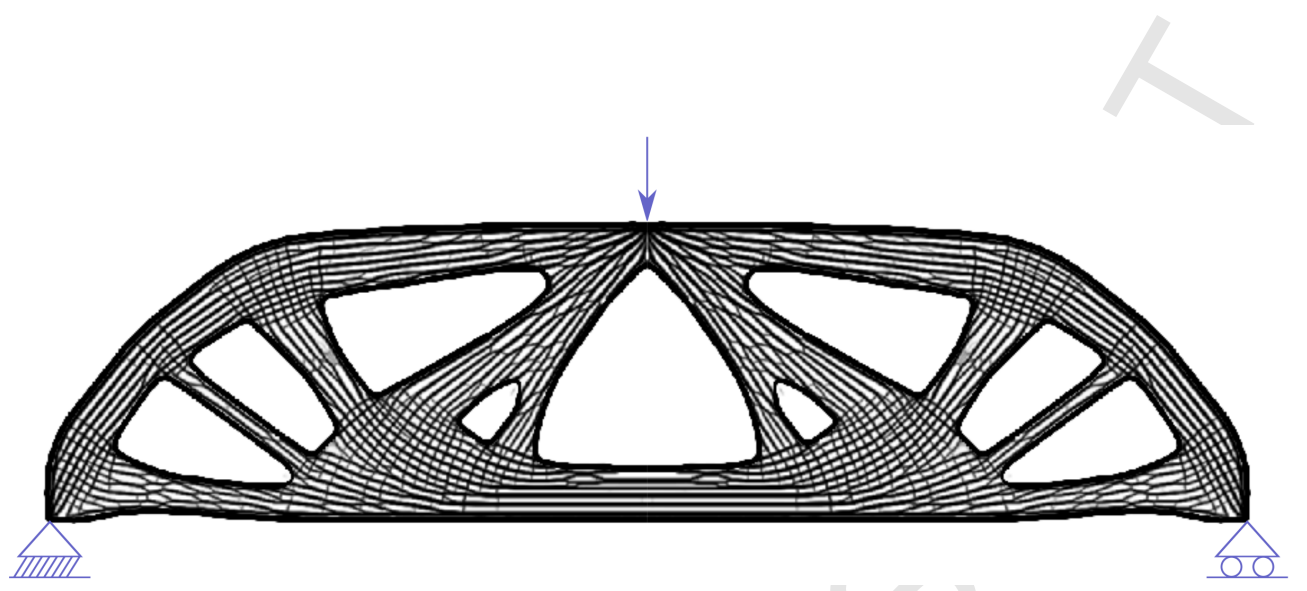

Figure 1: A shell-infill composite obtained from optimizing a simply supported beam.

by Lazarov and Wang in [22]. Our method involves and justifies the use of multiple filtering steps ( four smoothing steps and three projections in particular ), in addition to the interpolation of two design fields and a gradient norm operator. Arguably, it sets a new extreme with respect to the number of the involved filtering operations - The most complicated combo of filters so far seems to be the four successive filters for the open-close operation suggested in [19]. Here the four filtering steps and their associated projections are used to control both macro and microstructures. Thus beyond proving the new capability of filtering schemes, our method also demonstrates the good scalability of multiple filtering in density-based optimization.

The remainder of this paper is organized as follows. Section 2 describes the shell-infill composite our method is aiming at. Section 3 presents the material model of shell-infill composites, considering the base, coating, and infill. Section 4 presents the optimization formulation, including local and global volume constraints and sensitivity analysis. Section 5 demonstrates the effectiveness of the proposed formulation on numerical examples. Section 6 concludes the paper.

\section{Problem Statement}

Before we rigorously define the optimization problem in the following sections, let us illustrate what optimized structures we are aiming at. The composites resulting from numerical optimization, without post-processing, shall fulfill a few geometric features. The optimized composite for a sample beam design problem is shown in Fig. 1. Specifically, the composite structure has a shell with a prescribed thickness $(t)$, and an infill with a prescribed local volume fraction $(\gamma)$ at each point in the infill. In the discretized setting, the localized volume fraction of element $e$ is defined as the number of solid elements over the total number of elements in a small neighbourhood around element $e$. In this example, the prescribed local volume fraction is 0.6.

In the topology optimization process, the shell, including its shape and topology, evolves while maintaining the thickness $t$. Concurrently evolving is the infill which spreads over the region defined by the (dynamically changing) shell. The infill evolves to non-uniformly distributed micro-structures, regulated by a constraint on local volume fractions. The concurrent evolution of shell-infill is governed by the objective for achieving the highest stiffness under prescribed external loads, while respecting a constraint on the global material volume. 
(a)

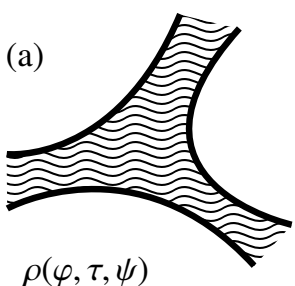

(b)

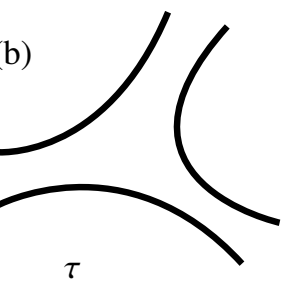

(c)

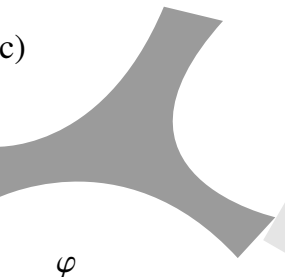

(d)

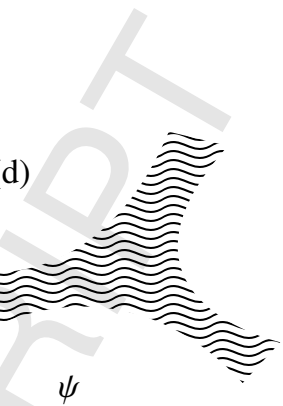

Figure 2: Conceptual illustration of shell-infill composites. The shell-infill structure (a) is composed of a solid shell (b) and a porous infill $(\mathrm{d})$. The shell and infill are related by a base region (c): The shell forms the boundary of the base region, while the infill falls into the base region. A wave infill pattern is used for the illustrative purpose.

\section{Material Model}

Figure 2 illustrates a conceptual composition of the shell-infill composite (a). It is composed of a solid shell (b) and a porous infill (d). These two sub-structures are related by a base region (c). The solid shell forms the interface (also called coating in the following) between base region and void, and the porous infill spreads across the entire base region. The physical density field of the shell-infill composite, $\rho(\varphi, \tau, \psi)$, is a function taking as input three intermediate fields, the base $(\varphi)$, the coating $(\tau)$, and the infill $(\psi)$.

The intermediate density fields are derived from two scalar fields of design variables, defined on the design domain $\Omega$. From the first field of design variables a two-step filtering process is applied to separate the base structure and the coating structure (Section 3.1). From the second field of design variables we obtain what can be called an enrichment field, by imposing constraints on a local volume measure (Section 3.2). The integration of intermediate fields into the final physical density field is realized by a material interpolation model (Section 3.3).

\subsection{Coating-Base Structure}

A two-step filtering process [10] is applied to derive the base and coating structures. As illustrated in the left of Fig. 3, the design field, $\mu$, is smoothed to get rid of checkerboard patterns, giving $\hat{\mu}$. The smoothed field is subsequently projected, leading to a sharp base structure, $\varphi=\overline{\hat{\mu}}$. The smoothing radius $\left(R_{1}\right)$ and projection parameters $\left(\eta_{1}\right.$ and $\left.\beta_{1}\right)$ indirectly control the length scale of the base region. The details of the filter implementation are postponed to Section 3.4.

To derive the coating layer, a second smoothing (with a filter radius $R_{2}$ smaller than $R_{1}$ ) is applied, giving $\hat{\varphi}$. This smoothing is necessary since the base density field $\varphi$ is non-smooth across the sharp edges, and the gradient therefore is not defined. The interface between the base region and void is defined by a gradient norm of the smoothed base field $\hat{\varphi}$,

$$
\|\nabla \hat{\varphi}\|_{\alpha} \equiv \alpha\|\nabla \hat{\varphi}\| .
$$

Here $\|\nabla \hat{\varphi}\|$ refers to the Euclidean norm of the spatial gradient of $\hat{\varphi} . \alpha$ is a normalization factor defined as the inverse of the maximum possible gradient norm of the field $\hat{\varphi}$. It can be analytically determined that $\alpha$ relates to $R_{2}$ as

$$
\alpha=\frac{R_{2}}{\sqrt{3}} .
$$

The shell thickness, $t$, of the projected sharp coating, $\tau$, is prescribed through the smoothing radius $R_{2}$ :

$$
R_{2}=\frac{\sqrt{3}}{\ln (2)} t \approx 2.5 t
$$




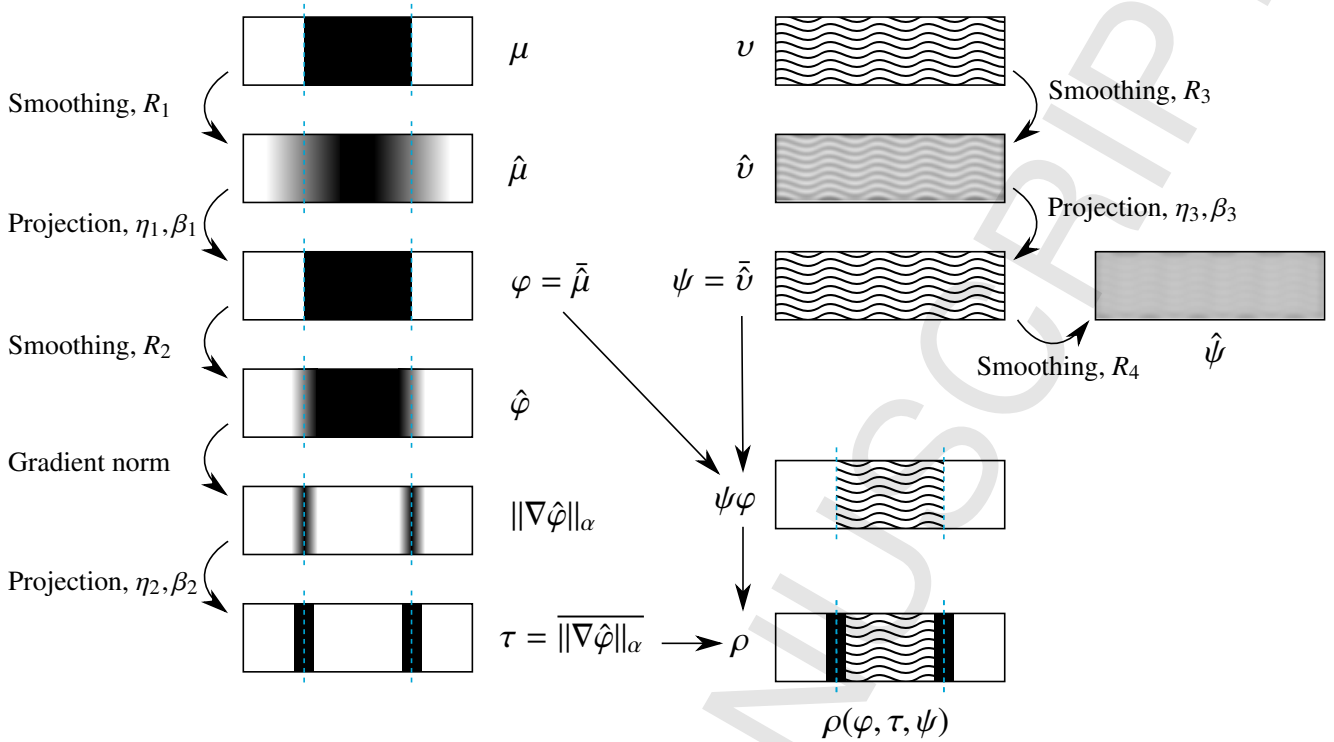

Figure 3: Left, top-down: The first design variable $\mu$ allows to identify the base region $\varphi$ and the coating $\tau$. The dashed lines indicate the interface as a visual reference. Top right: The second design variable $v$ defines the infill field $\psi$, and the local material measure $\hat{\psi}$. A wave infill pattern is used for the illustrative purpose. The actual infill field evolves under constraints imposed on $\hat{\psi}$. Bottom right: The intermediate field $\varphi, \tau$, and $\psi$ are interpolated to obtain the final physical density field $\rho$.

Analytical derivations are omitted here but the details can be found in [10].

\subsection{Enrichment Field and Local Volume Measure}

Rather than prescribing a fixed infill pattern which limits design flexibility [10], the infill evolves from a second design field and is decided by the numerical optimization process. The enrichment field, as we call the infill field, will be superimposed on the plain base region to create fine structures.

As illustrated in the top right of Fig. 3, the second design field, $v$, is smoothed (giving $\hat{v}$ ) and projected, resulting in a sharp infill field, $\psi=\overline{\hat{v}}$. Similar to the first design field, the smoothing is to get rid of checkerboard patterns. The smoothing radius $\left(R_{3}\right)$ and projection parameters $\left(\eta_{3}\right.$ and $\beta_{3}$ ) indirectly control the length scale, this time, of the infill details.

Upon the infill field $(\psi)$, a further smoothing is applied, giving $\hat{\psi}$. This smoothing is meant to quantify the local material accumulation. By imposing an upper bound on this measure, material is prevented from forming large solid regions, which thus effectively leads to a porous distribution [8]. The constraint will be explained in Section 4.1. For $\hat{\psi}_{e}=1$ (resp. $\left.\hat{\psi}_{e}=0\right)$ it means that all the elements located in the filtering region $\mathbb{N}_{e}$ are black (resp. white). The filtering region for element $e$ is defined as

$$
\mathbb{N}_{e}=\left\{i \mid\left\|x_{i}-x_{e}\right\|_{2} \leq R_{4}\right\}
$$

A value of $\hat{\psi}_{e}$ between 0 and 1 means that some elements in $\mathbb{N}_{e}$ are black and some others are white, yet it does not specify which particular elements are black (or white). This allows flexibility for the infill evolving to increase the structural stiffness. 


\subsection{Material Interpolation}

\subsubsection{Density Interpolation}

After getting the intermediate density fields $(\varphi, \tau$, and $\psi)$, the physical density $\rho$ is defined as an interpolation:

$$
\rho(\varphi, \tau, \psi)=m^{0}[\psi \varphi+(1-\psi \varphi) \tau],
$$

where $m^{0}$ is the mass density of the base material. For simplicity let us assume $m^{0}=1$ as in the standard SIMP. The interpolated density $\rho$ has values in the interval $[0,1]$, since $\varphi, \tau$, and $\psi$ obey the same bound. Considering $\psi \varphi$ as an integral part, the interpolation of the three fields can be interpreted as an interpolation of $\psi \varphi$ and $\tau$. This type of interpolation has been studied in [23].

We examine this interpolation by considering three extreme cases where the base and coating fields converge to a discrete $0 / 1$ solution.

- In the sharp interface defined by $\tau=1$ in the coating field, the density interpolation (Eq. 5) reduces to

$$
\rho(\varphi, 1, \psi)=\psi \varphi+(1-\psi \varphi) \cdot 1=1
$$

- In the base region enclosed by the sharp interface, i.e., defined by $\varphi=1$ and $\tau=0$, the density interpolation reduces to

$$
\rho(1,0, \psi)=\psi \cdot 1+(1-\psi \cdot 1) \cdot 0=\psi
$$

In other words, in the base region the enrichment infill field $\psi$ will be the output.

- In the void region outside the sharp interface, i.e., defined by $\varphi=0$ and $\tau=0$, the density interpolation reduces to

$$
\rho(0,0, \psi)=\psi \cdot 0+(1-\psi \cdot 0) \cdot 0=0
$$

\subsubsection{Stiffness Interpolation}

In line with the density interpolation from three density fields, the stiffness is interpolated from three stiffness fields, each of which resembles the standard SIMP stiffness for one density field,

$$
E(\varphi, \tau, \psi)=E^{0}\left[\psi^{p} \varphi^{p}+\left(1-\psi^{p} \varphi^{p}\right) \tau^{p}\right],
$$

where $E^{0}$ is the stiffness of the base material. The penalization parameter $p=3$ is the same for the three fields. The composite stiffness interpolation differs from the standard SIMP interpolation where the stiffness is defined as an explicit function of the physical density $\rho$.

For the three extreme cases (the coating, the base, and the void regions) the stiffness function reduces, respectively, to

$$
\begin{aligned}
& E(\varphi, 1, \psi)=E^{0}, \\
& E(1,0, \psi)=\psi^{p} E^{0}, \\
& E(0,0, \psi)=0 .
\end{aligned}
$$


The above interpolations assume that the infill and shell have the same material with a mass density of $m^{0}=1$ and a stiffness of $E^{0}$. To allow the possibility of using a different material for the infill, a scaling factor $\lambda_{m}$ for mass density and a factor $\lambda_{E}$ for stiffness are applied to the infill field. The interpolations become

$$
\begin{aligned}
& \rho(\varphi, \tau, \psi)=\lambda_{m} \psi \varphi+\left(1-\lambda_{m} \psi \varphi\right) \tau \\
& E(\varphi, \tau, \psi)=E^{0}\left[\lambda_{E} \psi^{p} \varphi^{p}+\left(1-\lambda_{E} \psi^{p} \varphi^{p}\right) \tau^{p}\right] .
\end{aligned}
$$

The scaling factors $\lambda_{m}$ and $\lambda_{E}$ are contained in the interval [0,1], meaning that the infill is (optionally) made of a lighter, and softer material than the shell material. We use this twomaterial formulation in optimization due to its generality.

This two-material formulation reduces to simpler forms in extreme cases. In case of $\lambda_{m}=1$ and $\lambda_{E}=1$, the two materials have identical mass and stiffness properties, i.e., reducing to the formulation of a single material. In case of $\psi=1$, i.e., prescribing a fully solid infill field, the two-material formulation reduces to the standard coating approach [10].

\subsection{Filters}

Two types of filters are commonly used in density-based topology optimization. A smoothing filter applies a convolution operator to smooth the density field. A projection filter thresholds grey scale values between 0 and 1 into white-or-black values, i.e., either 0 or 1 .

\subsubsection{Smoothing}

Smoothing filters are applied four times for different purposes. The smoothing of $\mu \rightarrow \hat{\mu}$ and $v \rightarrow \hat{v}$ gets rid of checkerboard patterns (i.e., regions of alternating black and white elements) resulting from numerical instabilities [24]. The smoothing of $\varphi \rightarrow \hat{\varphi}$ is to identify the interface of the base structure. The smoothing of $\psi \rightarrow \hat{\psi}$ is to quantify the local material accumulation.

We use the so-called PDE-filter based on a Helmholtz-type partial differential equation [25]. The PDE-filter is efficient for handling large filter radii. Hence, it is beneficial in the current work, since the length scale of the base structure necessitates a large filter radius, e.g., $R_{1}=24$ in some of test cases. The smoothed density field is implicitly defined as a solution to the Helmholtz PDE:

$$
-r^{2} \nabla^{2} \hat{x}+\hat{x}=x, x \in\{\mu, \varphi, v, \psi\} .
$$

The scalar-valued $r$ is a length scale parameter. Its value is determined by the filter radius, $R$, in the standard filtering technique by a convolution operator,

$$
r=\frac{R}{2 \sqrt{3}} .
$$

The filter radius $R$ indirectly controls the length scale of the respective field. The four radii in the four smoothing steps generally have different values.

The PDE-filter in $\psi \rightarrow \hat{\psi}$ serves the same purpose as the convolution filter in the original infill approach [8]: to quantify the local material accumulation. They differ slightly on the weighting factors: The convolution filter in the original infill approach has a constant weighting factor, while the PDE-filter has a weighting factor gradually decreasing along the outwards radial direction. We consistently use the PDE-filter for the four smoothing steps in our current implementation. 


\subsubsection{Projection}

Smoothing operators create density values between 0 and 1 . Such grey values are thresholded to obtain black-and-white designs by projection methods [18, 19]. Differentiable projections are used to facilitate gradient-based numerical optimization. We use the projection function proposed in [14]:

$$
\bar{x}_{i}=\frac{\tanh (\beta \eta)+\tanh \left(\beta\left(x_{i}-\eta\right)\right)}{\tanh (\beta \eta)+\tanh (\beta(1-\eta))}, x \in\left\{\hat{\mu},\|\nabla \hat{\varphi}\|_{\alpha}, \hat{v}\right\} .
$$

$\mathrm{m}$ The projection is parametrized by two values, the 'sharpness' value, $\beta$, and the threshold value, $\eta \in[0,1]$. The differentiable function approaches a discontinuous step function in the limit of $\beta \rightarrow \infty$. A parameter continuation process starting from a small $\beta$ value is applied to improve convergence behaviour, in contrast to directly starting with a very large $\beta$ value.

Denote the projected field for $\eta=0.5$ as a reference field. A larger threshold value of $\eta>0.5$ results in an eroded field compared to the reference field. As $\eta$ approaches 1 the projection becomes similar to the modified Heaviside step function [19]. A smaller threshold value of $\eta<0.5$ results in a dilated field compared to the reference field. As $\eta$ approaches 0 the projection becomes similar to the Heaviside step function [18].

In the three projection steps resulting in the physical density field, we choose the same threshold value of $\eta_{1,2,3}=0.5$. We note that an eroded projection by $\eta>0.5$ can be applied to control the length scale. This will be discussed in Section 4.3 where we explore this possibility to control the length scale of the base structure.

\section{Shell-Infill Optimization}

We consider a standard compliance minimization problem. However, besides a constraint on the global volume as in standard topology optimization, a constraint is imposed on the local volume measure obtained from the second design field. The optimization problem is defined as follows.

$$
\begin{array}{cl}
\min _{\mu, v} & c=\boldsymbol{U}^{T} \boldsymbol{K} \boldsymbol{U}, \\
\text { s.t. } & \boldsymbol{K} \boldsymbol{U}=\boldsymbol{F}, \\
& l(\boldsymbol{v}) \leq 0, \\
& g(\boldsymbol{\mu}, \boldsymbol{v}) \leq 0, \\
& \mu_{e}, v_{e} \in[0,1], \forall e .
\end{array}
$$

Here $c$ is the compliance. $\boldsymbol{U}, \boldsymbol{K}$, and $\boldsymbol{F}$ are displacement vector, stiffness matrix, and force vector, respectively. The functions $l$ and $g$ represent local and global volume constraint, respectively. $\boldsymbol{\mu}$ and $\boldsymbol{v}$ are vectors of element values in the design fields $\mu$ and $v$, respectively. The subscript $e$ indicates an element in the design domain. The global stiffness matrix $\boldsymbol{K}$ is assembled from element stiffness matrix $\boldsymbol{k}_{e}$, which is defined as:

$$
\boldsymbol{k}_{e}=E_{e}\left(\varphi_{e}, \tau_{e}, \psi_{e}\right) \boldsymbol{k}_{0}
$$

where $\boldsymbol{k}_{0}$ is the element stiffness matrix for an element with unit Young's modulus. To prevent the global stiffness matrix from becoming singular, a small minimum stiffness $\lambda_{E \text {,min }} E^{0}$ is used to represent void element. This modifies the term $\lambda_{E} \psi^{p} \varphi^{p}$ in Eq. 14 to $\lambda_{E \text {, min }}+\left(\lambda_{E}-\lambda_{E, \min }\right) \psi^{p} \varphi^{p}$. 
In the following we discuss the local volume constraint (Section 4.1), the global volume constraint (Section 4.2), the application of a length scale (Section 4.3), and the sensitivity analysis (Section 4.4). The optimization problem is solved based on sensitivities using the method of moving asymptotes (MMA) [26].

\subsection{Local Volume Constraints (Infill)}

Local volume constraints regulate the local (infill) material distribution. For each element in the second design field, a constraint is imposed,

$$
\hat{\psi}_{e} \leq \gamma, \forall e
$$

with $\gamma$ being the upper bound on the local volume fraction. The upper bound is a fixed parameter specified by the designer. For a prescribed value smaller than 1 , the local volume constraint prevents material from forming large solid parts. For instance, $\gamma=0.5$ leads to a material distribution where, in the neighbourhood of each element, at most $50 \%$ elements can become solid. The neighbourhood is defined by the filter radius $R_{4}$. This upper bound thus controls the local porosity.

The number of local volume constraints equals the number of elements. To facilitate efficient numerical optimization, we use the $p$-norm to approximate the maximum value in $\hat{\psi}$,

$$
\|\hat{\psi}\|_{p_{n}}=\left(\sum_{e} \hat{\psi}_{e}^{p_{n}}\right)^{1 / p_{n}} \approx \max _{\forall e}\left(\hat{\psi}_{e}\right) .
$$

Here $p_{n}$ denotes the $p$-norm parameter, to distinguish from the penalization $p$. This approximation reduces constraints Eq. 24 to a single, differentiable constraint,

$$
\|\hat{\psi}\|_{p_{n}} \leq \gamma
$$

As $p_{n}$ goes to infinity, the approximation error between the $p$-norm and max function becomes zero. To account for the approximation error when the value of $p_{n}$ is not infinitely large, we modify the aggregated constraint to

$$
\left(\sum_{e} \hat{\psi}_{e}^{p_{n}}\right)^{1 / p_{n}} \leq\left(\sum_{e} \gamma^{p_{n}}\right)^{1 / p_{n}},
$$

yielding the local volume constraint,

$$
l(\boldsymbol{v})=\left(\frac{1}{n} \sum_{e} \hat{\psi}_{e}^{p_{n}}\right)^{1 / p_{n}}-\gamma \leq 0,
$$

where $n$ is the number of elements.

\subsection{Global Volume Constraint}

The global volume is accumulated from the physical density field $\rho$. A straightforward global volume constraint takes the form

$$
\sum_{\forall e} \rho_{e} v_{e} \leq V^{*},
$$

where $v_{e}$ is the (constant) element volume, and $V^{*}$ is the maximum allowed volume. 

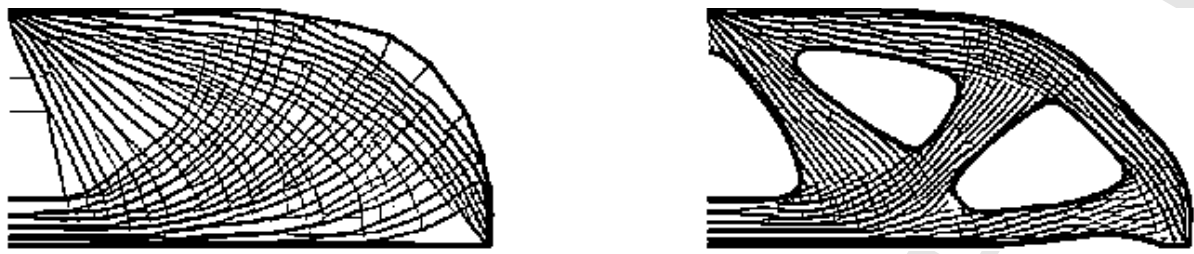

Figure 4: Left: The shell-infill composite obtained with the global volume constraint Eq. 29. Multiple large void parts appear in the base region, counteracting the intent to have distributed infill. Right: Composite obtained with the updated global volume constraint Eq. 31. Infill spreads over the entire base, making a clearly distinguishable shell-infill composite. The compliance is 98.2 (left) and 108.0 (right).
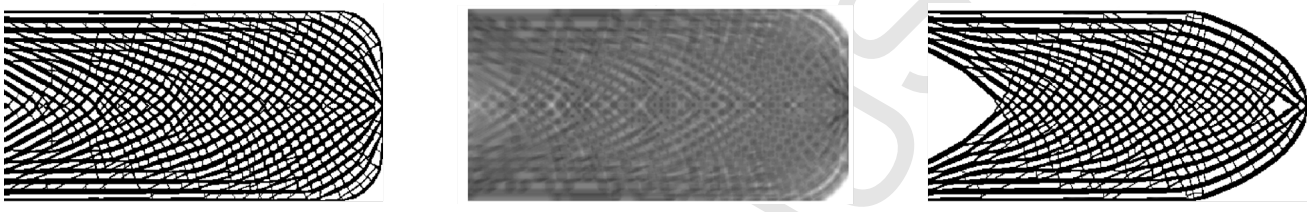

Figure 5: Left: The infill $(\psi)$ obtained with an upper bound $\gamma=0.6$ as local volume constraints, assuming the entire design space is a prescribed base region. No global volume constraint is imposed. Middle: The field of local volume measure $\hat{\psi}$. The local volume measures reach the upper bound $\gamma$ in the entire base region. Right: When a global volume constraint of ratio 0.5 is imposed, the infill shrinks to satisfy this constraint, leaving large void parts in the base region.

However, experimental tests show that when combined with local volume constraints for the infill, this global volume formulation leads to unintended structures. See Fig. 4 (left) for instance. Inside the base region, there are large void parts. These large voids counteract the design intention to have distinct shell-infill structures where the infill spreads over the entire base region enclosed by the shell.

To explain this, let us consider the simple case where the design space is a prescribed base region, i.e., the standard infill approach [8]. When the local volume constraints are imposed while the global volume constraint is inactive, the infill will spread over the entire base region (see Fig. 5 left): At each element, the local volume measure will reach the prescribed upper bound (see Fig. 5 middle for the field $\hat{\psi}$ ). This is in line with compliance minimization: The optimal structure always tries to exploit the full amount of material. If the global volume allowance for this region is smaller than necessary to spread the infills, (unintended) large void parts will have to be created (see Fig. 5 right).

Since the amount of base region in the concurrent optimization is not known a priori, the idea is that the allowed volume for infill shall be proportional to the area of the base region, rather than merely the area occupied by the sparse infill as in Eq. 29. In particular, assuming that every element takes the grey value of the upper bound $\gamma$, the physical density field becomes

$$
\dot{\rho}(\varphi, \gamma, \tau)=\lambda_{m} \gamma \varphi+\left(1-\lambda_{m} \gamma \varphi\right) \tau \text {. }
$$

The updated global volume constraint, in its normalized version, is

$$
g(\mu, \gamma)=\left(\sum_{e} \rho_{e} v_{e}\right) / V^{*}-1 \leq 0
$$

As can be seen on the right of Fig. 4, the updated global volume constraint serves the purpose of having infill spread over the base region. This updated global volume constraint is conserva- 


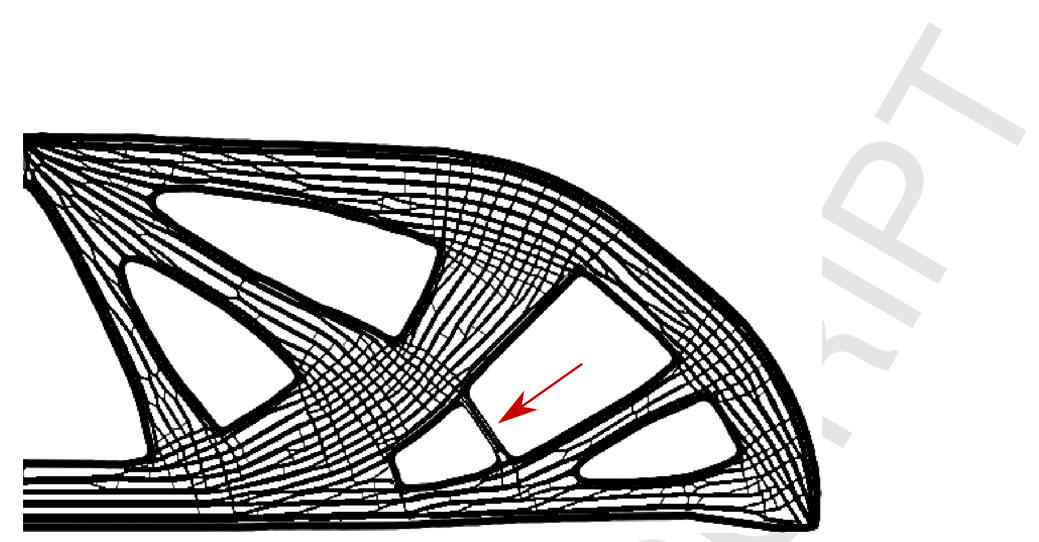

Figure 6: The shell-infill composite degenerates to a partial shell segment enclosing no infill, indicated by the arrow.

tive, in the sense that the global volume from the real physical density $\rho$ is smaller than that from the approximated density $\rho$, i.e.,

$$
\sum_{\forall e} \rho_{e} v_{e} \leq \sum_{e} \rho_{e} v_{e} .
$$

The two volumes become equal when the infill field takes the upper bound $\gamma$ for every element. As the infill converges to a discrete $0 / 1$ solution, the left becomes smaller. Consider the simple case of Fig. 5 where the entire design space is a prescribed base region. An upper local volume bound $\gamma=0.6$ leads to a global volume ratio of 0.53 (Fig. 5 left).

\subsection{Length Scale}

Length scale of the base is an additional option to help achieving distinct shell-infill composites. When the width of the base region is small, shell-infill locally degenerates to a shell enclosing no infill (See Fig. 6 for an example). To prevent this, we adapt the robust formulation $[14,27]$ to ensure a minimum length scale of the base. The robust formulation applies an erosion and a dilation projection, along with the intermediate projection. The projection parameter has been explained in Section 3.4.2, Eq. 17. By optimizing the structural performance on the worst scenario among the three projections, a minimum length scale on the solid and void phase can be ensured, resulting from the erosion and dilation projection, respectively.

In the context of compliance minimization, the worst case (i.e., the highest compliance value) among the three projections is the density field associated with erosion, since it uses the least amount of material. In the context of shell-infill optimization, while minimizing this worst case indeed ensures a length scale of the base, the coating at some locations is not clearly defined. An example is shown in Fig. 7 (top) for the standard coating approach, i.e., the infill constraint is not yet imposed for simplicity. The left and right are the coating-base structures corresponding to the intermediate and erosion projection, respectively. The coating in the intermediate projection (a) does not contribute in any way to the objective function, since the objective considers only the erosion projection which has a larger compliance value.

To encourage the appearance of a well-defined coating in the intermediate projection, the idea is to consider both the erosion projection and the intermediate projection in the robust formulation. Specifically, the following objective is minimized,

$$
c=\omega \boldsymbol{U}^{T} \boldsymbol{K} \boldsymbol{U}+(1-\omega) \boldsymbol{U}^{e T} \boldsymbol{K}^{e} \boldsymbol{U}^{e},
$$

where $\omega$ is a weighting factor, and the superscript $e$ in the second term indicates the eroded version. Figure 7 (bottom) demonstrates that the coating in the intermediate version is restored. 


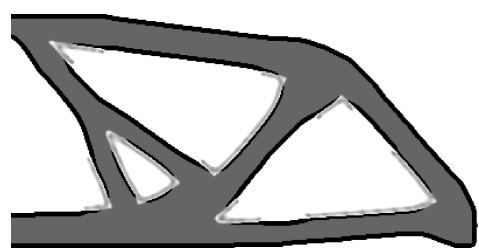

(a) $c=173.94$

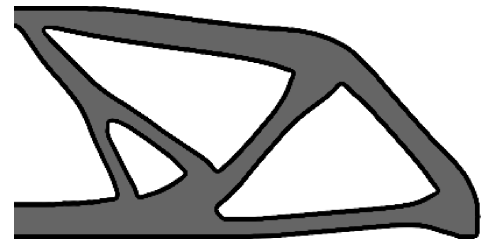

(c) $c=162.18$

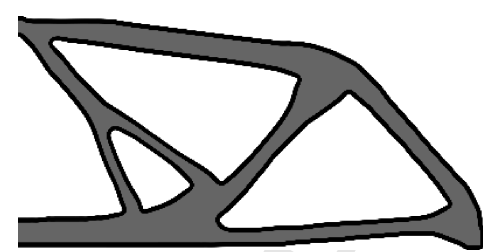

(b) $c=180.39$

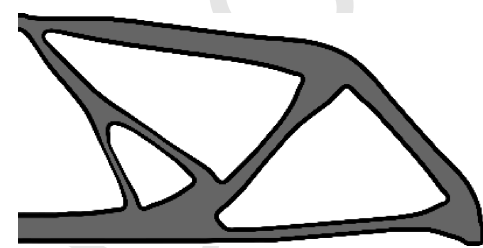

(d) $c=183.64$

Figure 7: Coating in the intermediate density field (a) is not well defined if only the compliance of the eroded version (b) is minimized in the objective function. Bottom: By minimizing both the intermediate and eroded versions, it encourages the appearance of the coating in the intermediate version (c). Both are tested with $v=0.3, \gamma=1, \lambda_{m}=0.6$, and $\lambda_{E}=0.33$.

We note that this combined objective function is an approximate approach to ensure length scale, since it involves a weighting factor. A small $\omega$ value leads to a shell which is not well defined, while a large $\omega$ value may lead to a structure violating the length scale. A continuation is applied in our test: $\omega$ is gradually increased from 0.1 to 0.8 , by an increment of 0.1 every 50 iterations.

\subsection{Sensitivity Analysis}

For solving the optimization problem defined by Eqs. 18-22 using the gradient-based MMA solver, the following sensitivities are needed:

$$
\frac{\partial c}{\partial \mu_{e}}, \frac{\partial c}{\partial v_{e}}, \frac{\partial l}{\partial \mu_{e}}, \frac{\partial l}{\partial v_{e}}, \frac{\partial g}{\partial \mu_{e}}, \text { and } \frac{\partial g}{\partial v_{e}} .
$$

All these sensitivities are elaborated in the following paragraphs.

(1) $\partial c / \partial \mu_{e}$ and $\partial c / \partial v_{e}$. From adjoint analysis the sensitivities of the compliance objective are:

$$
\frac{\partial c}{\partial x_{e}}=-\boldsymbol{U}^{T} \frac{\partial \boldsymbol{K}}{\partial x_{e}} \boldsymbol{U}=-\sum_{i} \frac{\partial E_{i}}{\partial x_{e}}\left(\boldsymbol{u}_{i}^{T} \boldsymbol{k}^{0} \boldsymbol{u}_{i}\right), x \in\{\mu, v\}
$$

To derive $\partial E_{i} / \partial \mu_{e}$ and $\partial E_{i} / \partial v_{e}$, we rewrite the stiffness interpolation (Eq. 14) by

$$
E(\varphi, \tau, \psi)=E^{0}\left[\lambda_{E} \psi^{p} \varphi^{p}+\left(1-\lambda_{E} \psi^{p} \varphi^{p}\right) \tau^{p}\right]=E^{0}[\underbrace{\lambda_{E} \psi^{p} \varphi^{p}}_{A_{E}}+\underbrace{\tau^{p}}_{B_{E}}-\underbrace{\lambda_{E} \psi^{p} \varphi^{p} \tau^{p}}_{C_{E}}] .
$$

Considering that $\psi(v)$ is independent of the first design variable $\mu$, and applying the chain rule and product rule, we arrive at

$$
\frac{\partial E_{i}}{\partial \mu_{e}}=E^{0}\left[\frac{\partial A_{E}}{\partial \varphi_{i}} \frac{\partial \varphi_{i}}{\partial \mu_{e}}+\frac{\partial B_{E}}{\partial \tau_{i}} \frac{\partial \tau_{i}}{\partial \mu_{e}}-\frac{\partial C_{E}}{\partial\left(\varphi_{i} \tau_{i}\right)}\left(\tau_{i} \frac{\partial \varphi_{i}}{\partial \mu_{e}}+\varphi_{i} \frac{\partial \tau_{i}}{\partial \mu_{e}}\right)\right],
$$


with $\partial A_{E} / \partial \varphi_{i}=\lambda_{E} \psi_{i}^{b} b \varphi_{i}^{b-1}, \partial B_{E} / \partial \tau_{i}=b \tau_{i}^{b-1}$, and $\partial C_{E} / \partial\left(\varphi_{i} \tau_{i}\right)=\lambda_{E} \psi_{i}^{b} b\left(\varphi_{i} \tau_{i}\right)^{b-1}$.

Similarly, considering $\varphi(\mu)$ and $\tau(\mu)$ are independent of $v$, this leads to

$$
\frac{\partial E_{i}}{\partial v_{e}}=E^{0}\left[\frac{\partial A_{E}}{\partial \psi_{i}} \frac{\partial \psi_{i}}{\partial v_{e}}-\frac{\partial C_{E}}{\partial \psi_{i}} \frac{\partial \psi_{i}}{\partial v_{e}}\right],
$$

with $\partial A_{E} / \partial \psi_{i}=\lambda_{E} \varphi_{i}^{p} p \psi_{i}^{p-1}$ and $\partial C_{E} / \partial \psi_{i}=\lambda_{E} \varphi_{i}^{p} \tau_{i}^{p} p \psi_{i}^{p-1}$.

(2) $\partial l / \partial \mu_{e}$ and $\partial l / \partial v_{e}$. The sensitivities of the local volume constraint with respect to the design variables has the form

$$
\frac{\partial l}{\partial x_{e}}=\sum_{i}\left(\sum_{j}\left(\frac{\partial l}{\partial \hat{\rho}_{j}} \frac{\partial \hat{\rho}_{j}}{\partial \rho_{i}}\right) \frac{\partial \rho_{i}}{\partial x_{e}}\right), x \in\{\mu, v\}
$$

with

$$
\frac{\partial l}{\partial \hat{\rho}_{j}}=\frac{1}{n}\left(\frac{1}{n} \sum_{e} \hat{\rho}_{e}^{p_{n}}\right)^{1 / p_{n}-1} \hat{\rho}_{j}^{p_{n}-1} .
$$

To derive $\partial \rho_{i} / \partial \mu_{e}$, Eq. 13 is rewritten as

$$
\rho(\varphi, \tau, \psi)=\lambda_{m} \psi \varphi+\left(1-\lambda_{m} \psi \varphi\right) \tau=\underbrace{\lambda_{m} \psi \varphi}_{A_{m}}+\underbrace{\tau}_{B_{m}}-\underbrace{\lambda_{m} \psi \varphi \tau}_{C_{m}} .
$$

Again, considering that $\psi(v)$ is independent of $\mu$, and applying the chain rule and product rule, it leads to

$$
\frac{\partial \rho_{i}}{\partial \mu_{e}}=\frac{\partial A_{m}}{\partial \varphi_{i}} \frac{\partial \varphi_{i}}{\partial \mu_{e}}+\frac{\partial B_{m}}{\partial \tau_{i}} \frac{\partial \tau_{i}}{\partial \mu_{e}}-\frac{\partial C_{m}}{\partial\left(\varphi_{i} \tau_{i}\right)}\left(\tau_{i} \frac{\partial \varphi_{i}}{\partial \mu_{e}}+\varphi_{i} \frac{\partial \tau_{i}}{\partial \mu_{e}}\right),
$$

with $\partial A_{m} / \partial \varphi_{i}=\lambda_{m} \psi_{i}, \partial B_{m} / \partial \tau_{i}=1$, and $\partial C_{m} / \partial\left(\varphi_{i} \tau_{i}\right)=\lambda_{m} \psi_{i}$.

For the design variable $v$, we have

$$
\frac{\partial \rho_{i}}{\partial v_{e}}=\frac{\partial A_{m}}{\partial \psi_{i}} \frac{\partial \psi_{i}}{\partial v_{e}}-\frac{\partial C_{m}}{\partial \psi_{i}} \frac{\partial \psi_{i}}{\partial v_{e}},
$$

with $\partial A_{m} / \partial \psi_{i}=\lambda_{m} \varphi_{i}$ and $\partial C_{m} / \partial \psi_{i}=\lambda_{m} \varphi_{i} \tau_{i}$.

(3) $\partial g / \partial \mu_{e}$ and $\partial g / \partial v_{e}$. The derivative of the total volume constraint with respect to the design variables is:

$$
\frac{\partial g}{\partial x_{e}}=\sum_{j} \dot{\rho}_{j} \sum_{i} \frac{\partial \rho_{i}}{\partial x_{e}}, x \in\{\mu, v\} .
$$

The modified density $\rho$ is independent of $v$, leading to

$$
\frac{\partial \rho_{i}}{\partial v_{e}}=0 .
$$

$\partial \rho_{i} / \partial \mu_{e}$ is a modified version of Eq. 42 by replacing $\psi$ therein with $\gamma$,

$$
\frac{\partial \dot{\rho}_{i}}{\partial \mu_{e}}=\lambda_{m} \gamma \frac{\partial \varphi_{i}}{\partial \mu_{e}}+\frac{\partial \tau_{i}}{\partial \mu_{e}}-\lambda_{m} \gamma\left(\tau_{i} \frac{\partial \varphi_{i}}{\partial \mu_{e}}+\varphi_{i} \frac{\partial \tau_{i}}{\partial \mu_{e}}\right) .
$$

(4) Elementary Derivatives. In above formulations, some elementary derivatives (e.g., $\partial \varphi / \partial \mu$, $\partial \tau / \partial \mu$, and $\partial \psi / \partial v)$ are not included here, but can be found in $[10,8]$. 

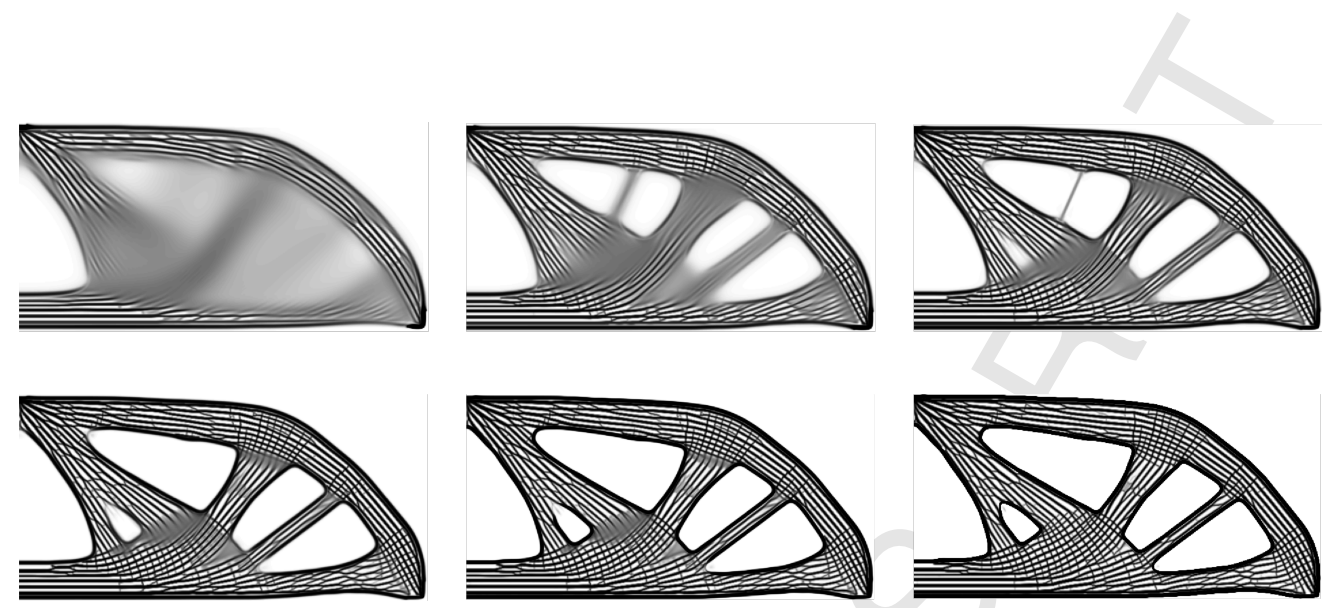

Figure 8: The shell-infill composite during the iterative optimization process. From left to right and top to down, the density distribution at the iterations of 100, 200, 300, 400, 500, 1000 .

\section{Results}

In this section we demonstrate the effectiveness of the proposed topology optimization for different examples. Variations of the optimized structures with respect to different design specification will be analyzed, to explain the consequence of the involved parameters.

The following parameters are the same for all examples. The constraints aggregation is calculated with a $\mathrm{p}$-norm value of $p_{n}=8$. A penalization $p=3$ is used in the stiffness interpolation (Eq. 9) for $\psi, \varphi$, and $\tau$. A projection threshold 0.5 is used for $\eta_{1}, \eta_{2}$, and $\eta_{3}$, while the eroded version of the base takes a value of 0.7. Parameter continuation is applied for the sharpness parameters. The coating sharpness is initialized with $\beta_{2}=8$ to get a sharp coating already from the beginning of the optimization, and doubled at every 100th iteration (or at convergence) until it is increased to 64 . The sharpness of base and infill starts from $\beta_{1,3}=1$, and is doubled together with $\beta_{2}$.

\subsection{Simply supported beam}

The first example is a simply supported beam, as shown in Fig. 1. Due to symmetry only one half of the design domain is simulated. The half design domain is discretized by square elements with a resolution of $300 \times 600$ to accommodate fine scale infill structures.

The problem is optimized using a total volume fraction of $40 \%$, and an upper local volume bound of 0.6. The four smoothing filter radii are $R_{1}=24$ for the base, $R_{2}=15$ for the coating (i.e., leading to a coating thickness of $t=6$ ),$R_{3}=2$ for the infill, and $R_{4}=8$ for quantifying the local volume fraction, all measured in terms of elements. The intermediate domains are extended from the design domain by a size of the largest filter radius $\left(R_{1}\right)$ in order to ensure the length scale on the boundary [28].

Figure 8 shows a sequence of the shell-infill composite during the design progression. As the optimization progresses, a clear shell-infill structure emerges from the grey density distribution. The optimized result shows two scales. The coarser scale defined by the shell looks familiar to standard topology optimization. Enclosed by the shell, the infill varies in different subregions, following the direction of the internal uni-axial stresses. This can be attributed to the fact that on the coarse scale as in standard topology optimization the optimized structure receives highly uni-axial stresses (compression or tension). At the joints connecting two bars, there are crossing 
Smoothing, $R_{1}$

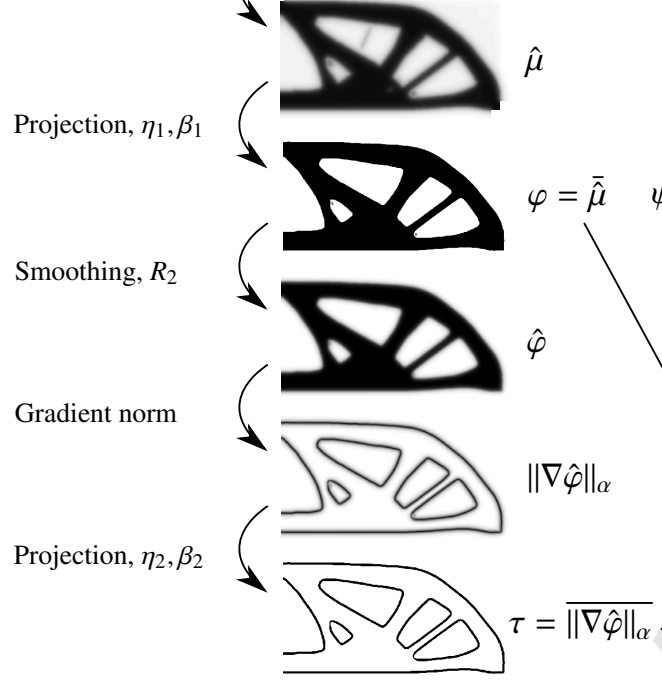

$v$
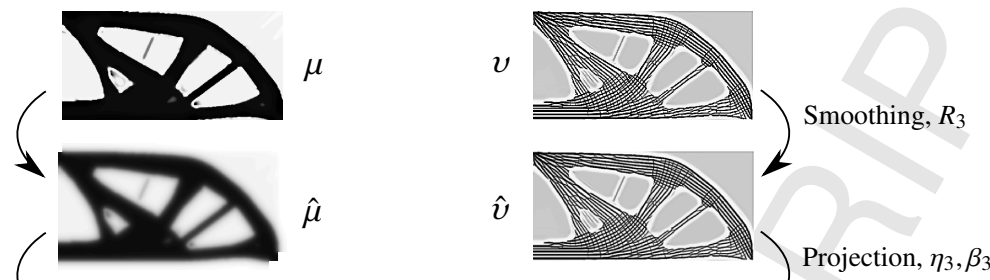

$\psi=\overline{\hat{v}}$

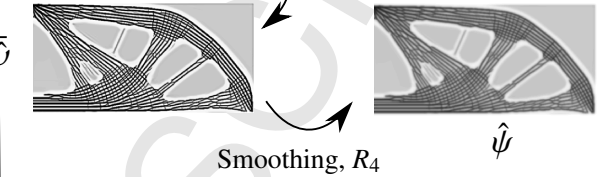

structures which naturally flow in from the infills in the adjacent branches, indicating bi-axial stresses in the crossings.

Figure 9 displays the intermediate density distributions involved in the shell-infill composition. The layout of this figure follows the schematic illustration in Fig. 3. On the left, from top to bottom, the density field of the first design variable is smoothed and projected to obtain a black-white base field $\varphi$. The base is smoothed, allowing its interface to be extracted by the gradient norm. The interface is further projected to obtain a sharp coating layer $\tau$. On the right, the density field of the second design variable evolves to a porous infill $\psi$. Due to the local volume constraints, the material forms small scale geometrical details in the region corresponding to the (black) base on the left. Since the enrichment field outside the base region does not affect the stiffness of the final composite structure, this outside region shows a uniform grey.

\subsection{Cantilever Beam}

The second numerical test is a cantilever beam. The design domain and boundary conditions are illustrated in Fig. 10. Due to symmetry only one half of the design domain is optimized, by using a discretization of $150 \times 500$ square elements. The resulting half structure is completed by its mirrored image to illustrate a complete structure.

The problem is optimized using a total volume fraction of $36 \%$. Different local volume upper bounds $(0.4 \sim 0.7)$ are applied to analyze the variations. The four filter radii in these tests are $R_{1}=16, R_{2}=10, R_{3}=2$, and $R_{4}=8$, all measured in terms of elements.

Figure 11 shows the four shell-infill composites with different infill porosities. As the local volume upper bound increases, the infill becomes denser. Since the same total volume constraint 


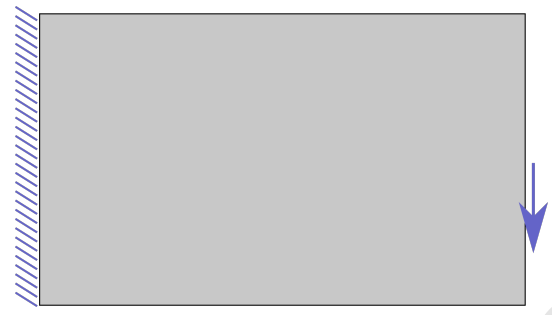

Figure 10: The design domain and boundary condition of the cantilever beam.

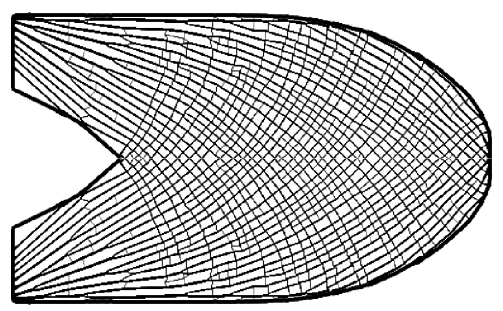

(a) $\gamma=0.4, v=0.285, c=181.43$

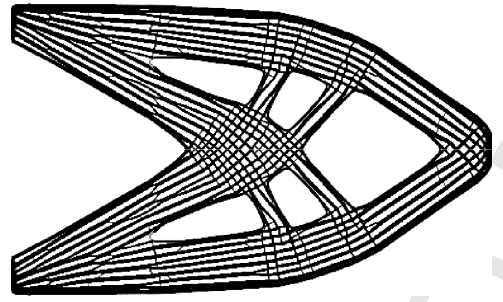

(c) $\gamma=0.6, v=0.337, c=125.40$

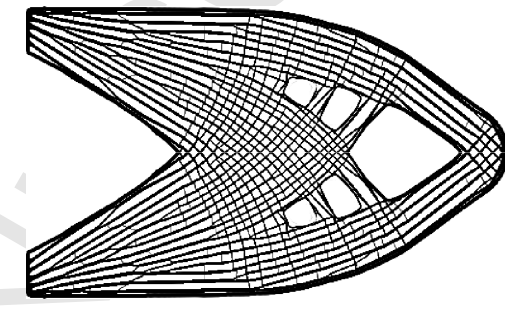

(b) $\gamma=0.5, v=0.309, c=144.69$

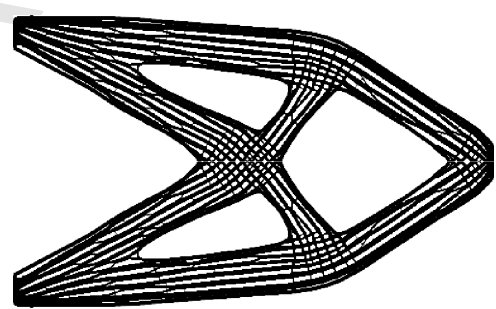

(d) $\gamma=0.7, v=0.353, c=115.97$

Figure 11: Cantilever beam example with different local volume upper bounds.

is applied, the denser infill results in a reduction of the base region. The compliance value also reduces as the infill becomes denser. To exclude the influence of the different volume values (which result from the infill approximation explained in Section 4.2), the compliance-volume ratios $(c / v)$ are calculated. In the order of an increasing infill fraction the ratios are 636.6, 468.3, 372.1 , and 328.5 , respectively.

\subsection{Multiple Loads}

Triangular-shaped (macro-)structures perform well for multiple, individually applied loads. Also, so-called rank-3 materials are optimal for microstructures subjected to multiple load cases. Hence, it is interesting to see whether such triangular-shaped structures also appear in the optimized infill when considering multiple load cases. Fig. 12 shows the design domain of a simply supported beam, upon which five vertical loads are applied individually. The objective function is the average of five compliances, each of which corresponds to an individual load. Fig. 13 shows the optimized shell-infill in this multi-load case. The multiple loads result in more variations of infill pattern than seen in the previous single load case problems. In particular, some triangular 


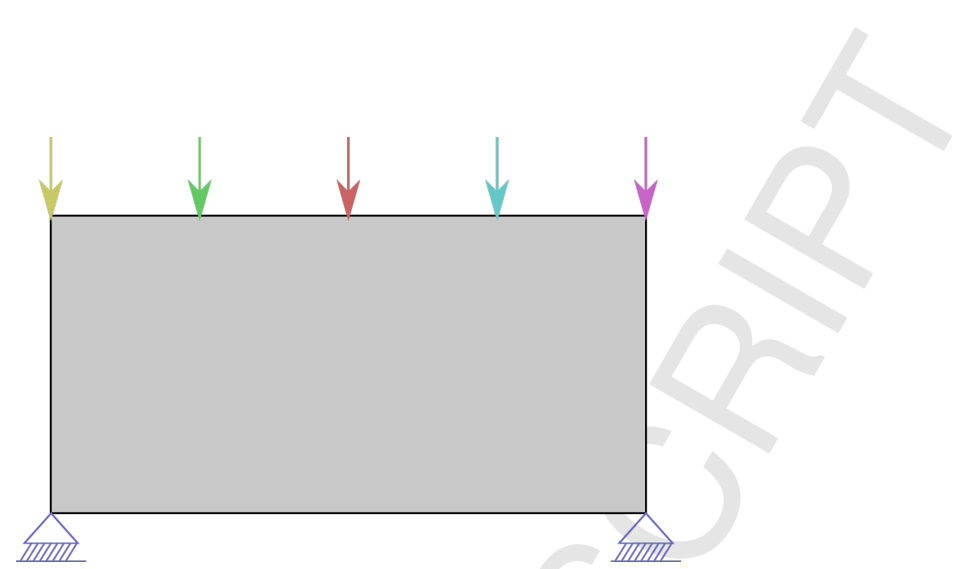

Figure 12: Design domain of a simply supported beam. The five loads have the same magnitude, and are applied individually.

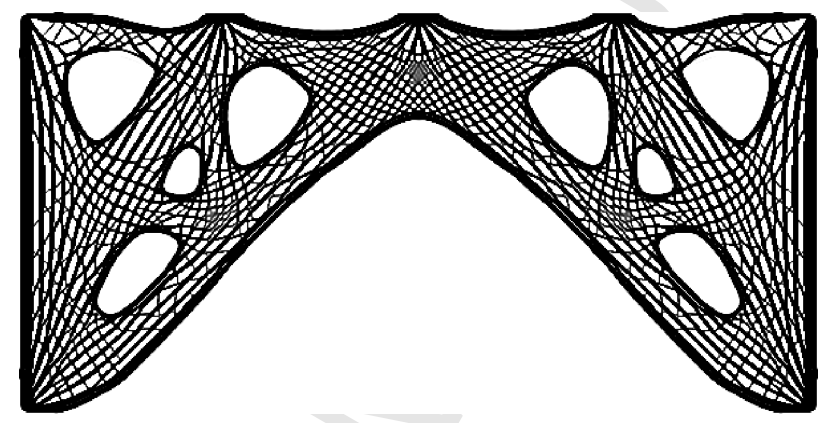

Figure 13: Optimized shell-infill for 5 individually applied loads.

sub-structures do appear and other regions have laminations that do not cross at right angles as was the rule in the single load case structures.

The domain has a resolution of $300 \times 600$ in terms of square finite elements. It is optimized with a maximum total volume fraction $36 \%$, a local volume upper bound $0.6, R_{1}=16, R_{2}=10$, $R_{3}=2$, and $R_{4}=8$.

\subsection{Comparison of Infills}

We compare the present optimization of shell-infill composites against the standard coating approach. The uniform infill in the standard coating approach can be interpreted as a prescribed, repetitive infill pattern. We first run the concurrent optimization with a local volume upper bound $\gamma=0.6$, and a total volume constraint of 0.4 . The obtained physical density field amounts to a total volume of 0.368 . With this total volume, we run the standard coating approach. Here, we assume the density of the uniform infill is 0.6 , the same value as the local upper bound in the shell-infill composite. The uniform infill is made of the same material as the coating. According to the Hashin-Shtrikman bounds for isotropic material [29], a density of 0.6 leads to a maximum effective stiffness of $1 / 3$. The two structures obtained using these two approaches are shown in Fig. 14. The numerical analysis suggests that the non-uniform infill performs much stiffer than the uniform infill for the same material volume.

The same conclusion can be drawn from a comparison performed on the cantilever beam model. The structures are shown in Fig. 15. The shell in these two designs has the same topology, 


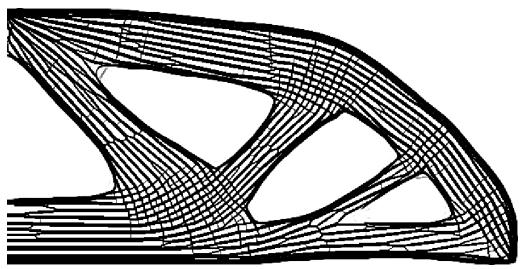

(a) $\gamma=0.6, \lambda_{m}=1.0, c=106.92$

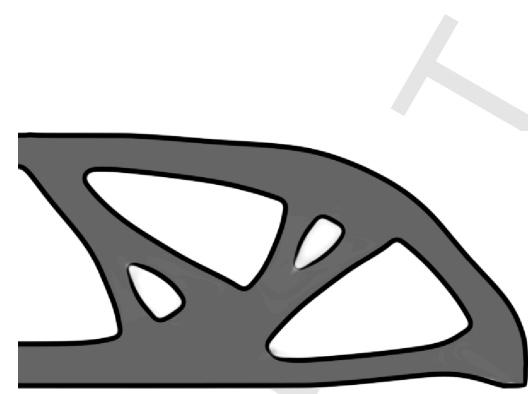

(b) $\gamma=1.0, \lambda_{m}=0.6, c=146.08$

Figure 14: Left: The shell-infill composite with an infill varying according to the stress distribution. Right: The infill is uniform. Both design use the same amount of total volume $(v=0.368)$.

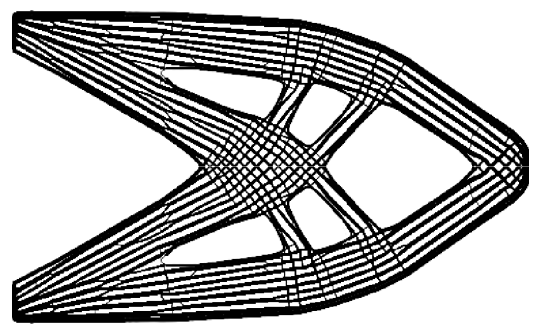

(a) $\gamma=0.6, \lambda_{m}=1.0, c=125.40$

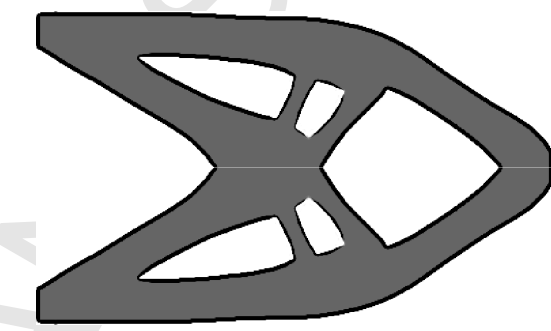

(b) $\gamma=1.0, \lambda_{m}=0.6, c=176.98$

Figure 15: A comparison of the non-uniform infill (left) and uniform infill (right) on the cantilever beam model. Both design use the same amount of total volume $(v=0.337)$.

while its contour varies slightly. The uniform infill (right) has a larger compliance value.

The improved performance of the optimized infill structures come at the cost of an expected decreased local buckling stability in the uni-axially loaded compression regions. Here single scale laminates will have low buckling stability but this may potentially be avoided by an anisotropic filtering approach suggested for the same reasons in our previous work [8].

\subsection{Two Materials}

In above tests we have assumed that the infill is made of the same material as the coating layer. The formulation (Eqs. 13 and 14) allows to use a different material representing the infill. Figure 16 shows an optimized cantilever beam using two different materials. The infill density field is grey, representing a relative density of $\lambda_{m}=0.6$. A relative stiffness of $\lambda_{E}=0.6$ is tested for the infill material. Note that when using two different materials, the relative stiffness is not restricted to the Hashin-Shtrikman bounds as in the standard coating approach, which interprets the infill as a structural pattern made of the coating material. In this example it can be observed that in the opening at the left hand side the shell is not well formed around the left corner. This is a side effect of the introduced length scale control, as discussed in Section 4.3. Further studies and modifications may alleviate the issue, but since it only appears sporadically we leave this issue for future studies. 


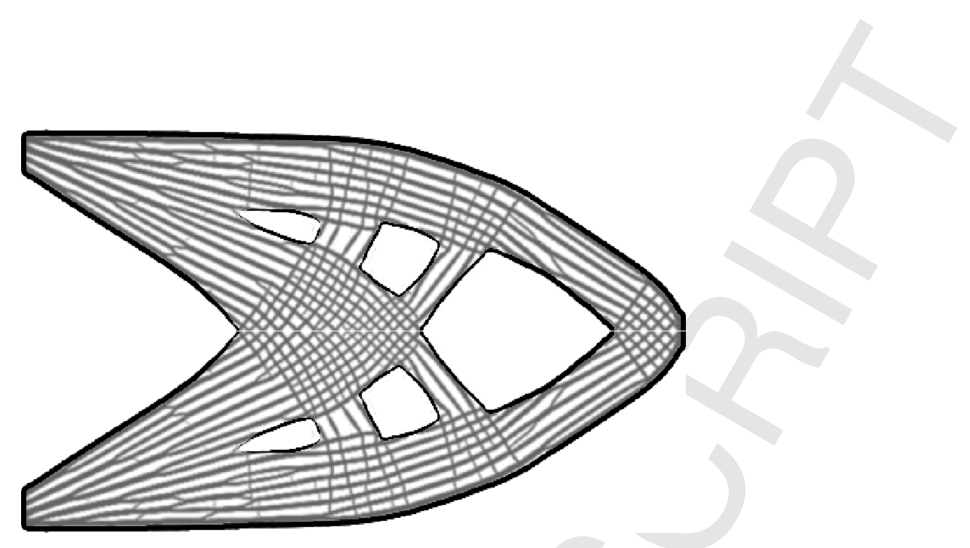

Figure 16: The shell-infill composite with a stiff material (black) for the shell, and a softer material (grey) for the infill.

\section{Conclusion}

We have presented a topology optimization method for evolving the shell and non-uniform infill concurrently. The composite maintains a prescribed shell thickness, and its interior is filled with non-uniform sparse structures up to a prescribed local volume fraction. The effectiveness of the proposed shell-infill interpolation model has been confirmed through numerical tests.

Our results demonstrate that optimized, non-uniform infill performs better than uniform infill for the same material volume. The optimized shell-infill shows some interesting geometric patterns. Elongated infill is found in the uni-axially loaded bars, while crossing infill can be observed at the joints connecting these bars. These results conform to, and can be explained by our understanding of optimal structures. With the increasing fabrication flexibility offered by additive manufacturing, it is expected that such tailor made structures will find many industrial applications.

Extending the shell-infill formulation from 2D to 3D is straightforward. The 3D results for the coating and the porous infill have been reported in [11] and [8], respectively. Closed-walled (infill) structures are efficient for stiffness. However, they might trap unsintered powders in powder-based fabrication. To steer closed-walled structures into truss-like structures, a possible solution is to restrict the local volume bound such that the locally allowable volume is not sufficient to create closed-walled structures [8].

Despite a rather complicated optimization model that includes four successive filter operations the algorithm is remarkably robust to parameter and geometric variations. Additional runs for other volume fractions and e.g., the classical MBB beam are easily solved and result in similar conclusions and are hence left out for space reasons.

\section{Acknowledgement}

The authors gratefully acknowledge the support from the H.C. Ørsted Postdoc Programme at the Technical University of Denmark, which has received funding from the People Programme (Marie Curie Actions) of the European Union's Seventh Framework Programme (FP7/20072013) under REA grant agreement no. 609405 (COFUNDPostdocDTU), and the support from the Villum foundation through the InnoTop VILLUM Investigator project. 


\section{References}

[1] M. P. Bendsøe, N. Kikuchi, Generating optimal topologies in structural design using a homogenization method, Computer methods in applied mechanics and engineering 71 (2) (1988) 197-224. doi : 10.1016/0045-7825 (88) 90086-2.

[2] M. P. Bendsøe, O. Sigmund, Topology optimization: theory, methods, and applications, Springer-Verlag Berlin Heidelberg, 2004.

[3] O. Sigmund, K. Maute, Topology optimization approaches, Struct. Multidiscip. Optim. 48 (6) (2013) $1031-1055$. doi: $10.1007 / \mathrm{s} 00158-013-0978-6$.

[4] J. D. Deaton, R. V. Grandhi, A survey of structural and multidisciplinary continuum topology optimization: post 2000, Struct. Multidiscip. Optim. 49 (1) (2014) 1-38. doi : 10.1007/s00158-013-0956-z.

[5] I. Gibson, D. W. Rosen, B. Stucker, Design for Additive Manufacturing, Springer US, Boston, MA, 2010 , pp. 299-332. doi:10.1007/978-1-4419-1120-9_11.

[6] W. Gao, Y. Zhang, D. Ramanujan, K. Ramani, Y. Chen, C. B. Williams, C. C. Wang, Y. C. Shin, S. Zhang, P. D. Zavattieri, The status, challenges, and future of additive manufacturing in engineering, Computer-Aided Design 69 (2015) 65-89. doi:10.1016/j.cad.2015.04.001.

[7] A. Clausen, N. Aage, O. Sigmund, Exploiting additive manufacturing infill in topology optimization for improved buckling load, Engineering 2 (2) (2016) 250 - 257. doi : 10.1016/J . ENG .2016.02.006.

[8] J. Wu, N. Aage, R. Westermann, O. Sigmund, Infill optimization for additive manufacturing - approaching bonelike porous structures, IEEE Transactions on Visualization and Computer Graphics PP (99) (2017) 1-1. doi: 10.1109/TVCG.2017.2655523.

[9] M. P. Bendsøe, O. Sigmund, Material interpolation schemes in topology optimization, Archive of Applied Mechanics 69 (9-10) (1999) 635-654. doi : 10.1007/s004190050248.

[10] A. Clausen, N. Aage, O. Sigmund, Topology optimization of coated structures and material interface problems, Computer Methods in Applied Mechanics and Engineering 290 (2015) 524 - 541. doi:10.1016/j.cma.2015. 02.011.

[11] A. Clausen, E. Andreassen, O. Sigmund, Topology optimization of 3d shell structures with porous infill, Acta Mechanica Sinica (0) 1.

[12] J. Guest, Imposing maximum length scale in topology optimization, Struct. Multidiscip. Optim. 37 (5) (2009) 463-473. doi:10.1007/s00158-008-0250-7.

[13] B. S. Lazarov, F. Wang, Maximum length scale in density based topology optimization, Computer Methods in Applied Mechanics and Engineering 318 (2017) 826-844. doi : 10.1016/j .cma.2017.02.018.

[14] F. Wang, B. S. Lazarov, O. Sigmund, On projection methods, convergence and robust formulations in topology optimization, Structural and Multidisciplinary Optimization 43 (6) (2010) 767-784. doi:10.1007/ s00158-010-0602-y.

[15] M. Langelaar, An additive manufacturing filter for topology optimization of print-ready designs, Struct. Multidiscip. Optim. (2016) 1-13doi : 10.1007/s00158-016-1522-2.

[16] X. Qian, Undercut and overhang angle control in topology optimization: a density gradient based integral approach, Int J Numer Meth Eng n/a-n/aNme.5461. doi : 10.1002/nme.5461.

[17] A. T. Gaynor, J. K. Guest, Topology optimization considering overhang constraints: Eliminating sacrificial support material in additive manufacturing through design, Struct. Multidiscip. Optim. 54 (5) (2016) 1157-1172. doi: 10.1007/s00158-016-1551-x.

[18] J. K. Guest, J. Prévost, T. Belytschko, Achieving minimum length scale in topology optimization using nodal design variables and projection functions, International Journal for Numerical Methods in Engineering 61 (2) (2004) 238254. doi:10.1002/nme.1064.

[19] O. Sigmund, Morphology-based black and white filters for topology optimization, Structural and Multidisciplinary Optimization 33 (4) (2007) 401-424. doi : 10.1007/s00158-006-0087-x.

[20] S. Xu, Y. Cai, G. Cheng, Volume preserving nonlinear density filter based on heaviside functions, Structural and Multidisciplinary Optimization 41 (4) (2010) 495-505. doi:10.1007/s00158-009-0452-7.

[21] J. Wu, C. C. Wang, X. Zhang, R. Westermann, Self-supporting rhombic infill structures for additive manufacturing, Computer-Aided Design 80 (2016) 32 - 42. doi : 10.1016/j.cad.2016.07.006.

[22] B. S. Lazarov, F. Wang, O. Sigmund, Length scale and manufacturability in density-based topology optimization, Archive of Applied Mechanics 86 (1) (2016) 189-218. doi : 10.1007/s00419-015-1106-4.

[23] O. Sigmund, Design of multiphysics actuators using topology optimization part ii: Two-material structures, Computer Methods in Applied Mechanics and Engineering 190 (4950) (2001) 6605 - 6627. doi:10.1016/ S0045-7825(01) 00252-3.

[24] A. Díaz, O. Sigmund, Checkerboard patterns in layout optimization, Structural optimization 10 (1) (1995) $40-45$. doi:10.1007/BF01743693. 
[25] B. S. Lazarov, O. Sigmund, Filters in topology optimization based on helmholtz-type differential equations, International Journal for Numerical Methods in Engineering 86 (6) (2011) 765-781. doi : 10.1002/nme. 3072.

[26] K. Svanberg, The method of moving asymptotes - a new method for structural optimization, International Journal for Numerical Methods in Engineering 24 (2) (1987) 359-373. doi : 10.1002/nme. 1620240207.

[27] O. Sigmund, Manufacturing tolerant topology optimization, Acta Mechanica Sinica 25 (2) (2009) 227-239. doi : 10.1007/s10409-009-0240-z.

[28] A. Clausen, E. Andreassen, On filter boundary conditions in topology optimization, Structural and Multidisciplinary Optimizationdoi : 10.1007/s00158-017-1709-1.

[29] Z. Hashin, S. Shtrikman, A variational approach to the theory of the elastic behaviour of multiphase materials, Journal of the Mechanics and Physics of Solids 11 (2) (1963) 127 - 140. doi :10.1016/0022-5096(63)90060-7. 


\section{Highlights}

- A novel method for optimizing shell-infill composites for additive manufacturing.

- The solid shell and the non-uniform, porous infill are optimized concurrently.

- The optimized infill spreads within the shell, and follows principal stress directions.

- Non-uniform infill can perform much stiffer than uniform infill.

- The method demonstrates the good scalability of multiple filtering in optimization. 